\title{
On the Levi-flats in Complex Tori of Dimension Two
}

\author{
By
}

\author{
Takeo OHSAwA*
}

\begin{abstract}
Compact Levi flat real analytic hypersurfaces in complex tori of dimension two are completely classified. The method is a combination of Siu's $\bar{\partial}$-regularity theory on weakly pseudoconvex domains, an updated variant of the $L^{2}$ estimate for the $\bar{\partial}$ operator originated from Berndtsson-Charpentier, and basic arguments in complex analytic geometry.
\end{abstract}

\section{$\S 0 . \quad$ Introduction}

Motivated by foliation theory, there arose an existence question for compact Levi flat hypersurfaces, or Levi-flats in short, in the complex $n$ dimensional projective space $\mathbb{P}^{n}$ (cf. [C]). After a pioneering work by A. LinsNeto [LN], Y.-T. Siu [S-1, S-2] settled the problem by showing that there exist no $C^{8}$ Leviflats in $\mathbb{P}^{n}$ if $n \geq 2$. The bound of regularity was later improved by A. Iordan [I] to $C^{4}$. In this development, particularly in Siu's work [S-2], the method of $L^{2}$ estimates for the $\bar{\partial}$-operator has been strengthened significantly. His idea was to refine the $L^{2}$ estimates on the domains with Levi flat boundary, to obtain a continuous solution to the $\partial \bar{\partial}$-equation restricted to the boundary. The required estimates are with respect to certain partial Sobolev norms associated to a one parameter group of metric preserving holomorphic automorphisms of $\mathbb{P}^{n}$. This idea was carried over with the aid of a Riemann type removability of singularities for the solutions of tangential Cauchy-Riemann equations.

Communicated by K. Saito. Received September 9, 2004. Revised November 11, 2004. 2000 Mathematics Subject Classification(s): Primary: 32V40; Secondary: 53C40.

* Graduate School of Mathematics, Nagoya University, Chikusaku Furocho, Nagoya 4648602, Japan.

e-mail: ohsawa@math.nagoya-u.ac.jp

(C) 2006 Research Institute for Mathematical Sciences, Kyoto University. All rights reserved. 
Here the singularities are situated along the set where the action of the one parameter group is not transverse to the Levi-flat. After the solution of such a regularity problem, nonexistence of $C^{8}$ Levi-flats in $\mathbb{P}^{n}$ naturally follows from the maximum principle for subharmonic functions. As for the ordinary $L^{2}$ estimates for the $\bar{\partial}$-operator, whose validity is very basic in Siu's proof, it is a direct consequence of the positivity of the Ricci curvature of $\mathbb{P}^{n}$. Accordingly, Siu's proof works also for the Hermitian symmetric spaces of compact type.

On the other hand, study of the Hartogs type extension theorem has been a natural alternative approach to the nonexistence question for the Levi-flats as in [LN]. In such a vein, G.M. Henkin and Iordan [H-I] has obtained a vanishing theorem for $L^{2} \bar{\partial}$-cohomology groups by exploiting a new technique of B. Berndtsson and P. Charpentier [B-C]. It applies to a class of smoothly bounded domains in Kähler manifolds with semipositive Ricci curvature.

In this circumstance, we would like to combine the method of Siu with an estimate of Berndtsson-Charpentier type to capture some knowledge about the Levi-flats in complex tori.

Our first observation in this direction is the following.

Theorem 0.1. Let $(X, \omega)$ be a homogeneous Kähler manifold of dimension two, and let $M \subset X$ be a $C^{5}$ Levi-flat. Then there exists no $C^{2}$ function $\varrho: X \backslash M \longrightarrow(-\infty, 0)$ which satisfies the following.

(0.1) There exists a neighbourhood $U \supset M$ and a positive constant $c$ such that $-i \partial \bar{\partial} \log (-\varrho)>c \omega$ holds on $U \backslash M$.

(0.2) There exists a nowhere vanishing $C^{2}$ function $u$ on a neighbourhood of $M$ such that $\varrho(x)=u(x)$ dist $(x, M)$ holds there. Here dist $(x, M)$ denotes the distance from $x$ to $M$.

Combining Theorem 0.1 with a computation in [M-O] we shall prove

Proposition 0.1. $\quad A C^{5}$ Levi-flat in a two dimensional complex torus contains a complex line segment.

Starting from this, we shall proceed to classify the Levi-flats in the tori, but restricting ourselves to the two dimensional cases only. Our main result is stated as follows.

Theorem 0.2. A real analytic Levi-flat in a two dimensional complex torus is either holomorphically flat or equivalent to a Levi scroll over an elliptic curve. (For the definitions, see $\S 4$.) 
This will be complemented by

Theorem 0.3. A two dimensional complex torus contains a Levi scroll if and only if it is fibered holomorphically over an elliptic curve.

\section{$\S 1$ Preliminaries}

First we shall recall an updated variant of a general theory of $L^{2}$ estimates for the $\bar{\partial}$-operator.

Let $(X, \omega)$ be a Kähler manifold of dimension $n$ and let $(D, \psi)$ be a hyperconvex domain in $X$. Here $\omega$ is a $d$-closed positive $(1,1)$ form on $X$ and $\psi$ is a strictly plurisubharmonic bounded exhaustion function on $D$.

We choose $\psi$ so that $\sup \psi=0$ and $\inf \psi>-1$.

Let $L_{(2)}^{p, q}(D)$ denote the space of $(p, q)$ forms on $D$ which are square integrable with respect to $\omega$, let $\bar{\partial}$ be the maximal closed extension of the complex exterior derivative of type $(0,1)$, and let $\bar{\partial}^{*}$ be the adjoint of $\bar{\partial}$.

We set for any $C^{2}$ real valued function $\varphi$

$$
\|u\|_{\varphi}^{2}=\int_{D} e^{-\varphi}|u|^{2} d V
$$

Here $d V$ denotes the volume form with respect to $\omega$.

For our $\psi$, the norm $\|u\|_{\psi}$ is equivalent to $\|u\|:=\|u\|_{0}$, since $\psi$ is bounded.

Let $\Lambda$ be the adjoint of the exterior multiplication by $\omega$. Then, for any $C^{\infty}$ compactly supported $(n, q)$ form $u$ on $D$, an inequality

$$
(i \partial \bar{\partial} \varphi \Lambda u, u)_{\varphi} \leq\|\bar{\partial} u\|_{\varphi}^{2}+\left\|\bar{\partial}_{\varphi}^{*} u\right\|_{\varphi}^{2}
$$

holds true. Here $d=\partial+\bar{\partial},(,)_{\varphi}$ denotes the inner product, and $\bar{\partial}_{\varphi}^{*}$ the adjoint of $\bar{\partial}$ with respect to the norm \|\|$_{\varphi}$ (see [O-1] for instance).

If $\varphi=\psi$ and $\partial D$ is $C^{2}$-smooth more is true. Namely

$$
(i \partial \bar{\partial} \psi \Lambda u, u)_{\psi} \leq\|\bar{\partial} u\|_{\psi}^{2}+\left\|\bar{\partial}_{\psi}^{*} u\right\|_{\psi}^{2}
$$

holds for any $u \in L_{(2)}^{n, q}(D) \cap \operatorname{Dom} \bar{\partial} \cap \operatorname{Dom} \bar{\partial}_{\psi}^{*}$ (cf. [H-1]). Here Dom $\bar{\partial}$ and Dom $\bar{\partial}_{\psi}^{*}$ denote the domains of $\bar{\partial}$ and $\bar{\partial}_{\psi}^{*}$, respectively.

Proposition 1.1. If $i \partial \bar{\partial} \psi>i(1+\varepsilon) \partial \psi \wedge \bar{\partial} \psi$ for some $\varepsilon>0$, then one can find $C>0$ such that

$$
(i \partial \bar{\partial} \psi \Lambda u, u) \leq C\left(\|\bar{\partial} u\|^{2}+\left\|\bar{\partial}^{*} u\right\|^{2}\right)
$$

holds for any $u \in L_{(2)}^{n, q}(D) \cap \operatorname{Dom} \bar{\partial} \cap \operatorname{Dom} \bar{\partial}^{*}$. 
Proof. Let $u \in L_{(2)}^{n, q}(D) \cap \operatorname{Dom} \bar{\partial} \cap \operatorname{Dom} \bar{\partial}^{*}$. Let $\chi:(-\infty, 0) \longrightarrow[0,1]$ be a $C^{\infty}$ function such that $\chi(t)=1$ for $t<-1 / 2$ and $\chi(t)=0$ for $t>-1 / 4$.

We set

$$
\varrho_{k}(x)=\chi(-k / \log (-\log (-\psi(x))))
$$

and $u_{k}=\varrho_{k} u$.

Let $\lambda:(-\infty, 0) \longrightarrow[0,1)$ be a $C^{\infty}$ convex increasing function such that $\lambda(t)=0$ for $t<-1$ and $\lambda^{\prime}(t)=3 / 2$ for $-1 / 2<t<0$.

Then we set

$$
\begin{aligned}
\mu_{k}(x) & =\lambda(-k / \log (-\log (-\psi(x)))) \\
\omega_{k} & =\omega+i \partial \bar{\partial} \mu_{k}
\end{aligned}
$$

and

$$
\psi_{k}=\psi+\mu_{k} .
$$

Let $\Lambda_{k}$ be the adjoint of exterior multiplication by $\omega_{k}$, let $\|\quad\|_{k}$ be the $L^{2}$ norm with respect to $\omega_{k}$ and $\psi_{k}$, and let $\bar{\partial}_{k}^{*}$ be the adjoint of $\bar{\partial}$ with respect to \|\|$_{k}$.

Then, for each $k$ we have

$$
\left(i \partial \bar{\partial} \psi_{k} \Lambda_{k} u_{k}, u_{k}\right)_{k} \leq\left\|\bar{\partial} u_{k}\right\|_{k}^{2}+\left\|\bar{\partial}_{k}^{*} u_{k}\right\|_{k}^{2} .
$$

Since $\omega_{k} \longrightarrow \omega$ and $\psi_{k} \longrightarrow \psi$ uniformly on compact subsets of $D$, and the lengths of $\bar{\partial} \rho_{k}$ and $\bar{\partial} \mu_{k}$ with respect to $\omega_{k}$ are uniformly bounded in $k$, by letting $k \longrightarrow \infty$ we obtain

$$
(i \partial \bar{\partial} \psi \Lambda u, u)_{\psi} \leq\|\bar{\partial} u\|_{\psi}^{2}+(1+2 / \varepsilon)\left\|\bar{\partial}^{*} u\right\|_{\psi}^{2}+(1+\varepsilon / 2)\left\|(\bar{\partial} \psi)^{*} u\right\|_{\psi}^{2} .
$$

Here $(\bar{\partial} \psi)^{*}$ denotes the adjoint of the exterior multiplication by $\bar{\partial} \psi$ from the left hand side.

Hence, by the assumption on $\partial \bar{\partial} \psi$ we obtain

$$
\frac{\varepsilon}{2(1+\varepsilon)}(i \partial \bar{\partial} \psi \Lambda u, u)_{\psi} \leq\|\bar{\partial} u\|_{\psi}^{2}+\left(1+\frac{2}{\varepsilon}\right)\left\|\bar{\partial}^{*} u\right\|_{\psi}^{2} .
$$

Therefore (1.3) holds for $C=\frac{1+\varepsilon}{\varepsilon}\left(1+\frac{2}{\varepsilon}\right)\left(\sup e^{-\psi}\right)\left(\sup e^{\psi}\right)$.

\section{$\S 2$. Siu's Theory for the $\bar{\partial}$-regularity}

We shall combine Proposition 1.1 with Siu's basic consideration on the $L^{2}$ estimates which guarantee, for the domains with Levi flat boundary of some type, the solvability of $\bar{\partial}$-equations with smoothness up to the boundary. 
For that, let us recall the outline of Siu's theory following [S-2] basically word for word.

Let $X$ be a connected complex manifold of dimension two equipped with a $C^{\infty}$ positive $(1,1)$ form $\omega$. $(X, \omega)$ will be refered to as a Hermitian surface. If $\omega$ is $d$-closed, $(X, \omega)$ will be called a Kähler surface. $(X, \omega)$ is said to be homogeneous if the group of $\omega$-preserving holomorphic automorphisms of $X$ acts transitively on $X$.

Definition (metric-preserving real vector fields). For a real vector field $\xi$ on $X$, we denote by $\mathrm{Lie}_{\xi}$ the Lie derivative with respect to $\xi$. We say that $\xi$ preserves $\omega$ if the Lie derivative of $\omega$ with respect to $\xi$ is identically zero.

Definition (thin sets). For a positive number $\varepsilon$ and a subset $Z$ of $X$, let $U_{\varepsilon}(Z)$ denote the $\varepsilon$-neighbourhood of $Z$. For any positive number $\kappa$, we say that $Z$ is thin of order at least $\kappa$ in $X$ if there exists a positive constant $C=C(Z)$ such that the volume of $U_{\varepsilon}(Z)$ with respect to $\omega$ is no more than $C \varepsilon^{\kappa}$. For a point $P_{0}$ of $X$ we say that $Z$ is locally thin at $P_{0}$ of order at least $\kappa$ in $X$ if there exists an open neighbourhood $W$ of $P_{0}$ in $X$ such that $Z \cap W$ is thin of order at least $\kappa$ in $X$.

Definition (Siu fields). $\quad$ Let $(X, \omega)$ be a Hermitian surface and let $M \subset$ $X$ be a Levi-flat of class $C^{q}$. A vector field $\xi$ on $X$ is said to be a Siu field for $M$ if the following conditions are satisfied.

(1) $\xi$ locally generates a germ of holomorphic isometries.

(2) $\xi$ is the real part of a holomorphic vector field $\tilde{\xi}$.

(3) There exist three subsets $Z_{j}(j=1,2,3)$ of $M$ satisfying the following conditions.

(3.i) $Z_{1}$ is a closed subset of $M$

(3.ii) $Z_{2}$ is a closed subset of $M \backslash Z_{1}$

(3.iii) $Z_{3}$ is a closed subset of $M$, and

(3.iv.a) $Z_{1}$ is thin of order at least three in $X$,

(3.iv.b) for every point $P_{0} \in Z_{2}$ the set $Z_{2}$ is locally thin at $P_{0}$ of order at least three in $X$,

(3.iv.c) for every point $P_{0} \in Z_{3} \backslash\left(Z_{1} \cup Z_{2}\right)$ the set $Z_{3}$ is locally thin at $P_{0}$ of order at least two in $X$, 
(3.iv.d) the vector field $\xi$ is not tangential to $M$ at every point of $M \backslash Z_{3}$, and

(3.iv.e) at every point of $M \backslash Z_{1}, \tilde{\xi}$ is not tangential to the leaf of the holomorphic foliation of $M$.

Since any vector field $\xi$ generating a group of holomorphic automorphism is automatically the real part of the holomorphic vector field $\tilde{\xi}=\xi-i J \xi$, one may ignore the condition (2) and replace (3.iv.e) by

(3.iv.f) at every point of $M \backslash Z_{1}, \xi$ is not tangential to the leaf of the holomorphic foliation of $M$.

In [S-2, Proposition 2.3], Siu proved that there exists a Siu field for any $C^{4}$ Levi-flat in $\mathbb{P}^{2}$. We shall slightly generalize it as follows.

Proposition 2.1. $\quad$ Let $(X, \omega)$ be a homogeneous Kähler manifold of dimension two, and let $M \subset X$ be a Levi-flat of class $C^{2}$. Then there exists a Siu field for $M$.

Proof. Let $P_{0} \in M$ be any point. By the homogeneity, there exist two holomorphic vector fields $\tilde{\xi}, \tilde{\eta}$ on $X$ such that $\tilde{\xi} \wedge \tilde{\eta}$ does not vanish at $P_{0}$. For any $(\alpha, \beta) \in \mathbb{C}^{2} \backslash\{(0,0)\}$ we put

$$
S_{(\alpha, \beta)}=\left\{v \in T_{M}^{\perp} \mid\langle\operatorname{Re}(\alpha \tilde{\xi}+\beta \tilde{\eta}), v\rangle=0\right\} .
$$

Here $T_{M}^{\perp}$ denotes the orthocomplement of $T_{M}$ in $T_{X} \mid M$ and $\langle$,$\rangle denotes the$ inner product.

Then, by Sard's theorem, there exists a subset $A \subset \mathbb{C}^{2}$ of Lebesgue measure zero such that, for any $(\alpha, \beta) \in \mathbb{C}^{2} \backslash A$, the image of $S_{(\alpha, \beta)}$ under the bundle projection $\pi: T_{M}^{\perp} \longrightarrow M$ is a $C^{1}$-smooth real curve on a neighbourhood of $P_{0}$. Hence, for any $(\alpha, \beta)$ outside some subset of $\mathbb{C}^{2}$ of Lebesgue measure zero, $\pi\left(S_{(\alpha, \beta)}\right)$ is a $C^{1}$-smooth curve outside the set $B=\left\{P \in M \mid(\tilde{\xi} \wedge \tilde{\eta})_{P}=0\right\}$. We note that a component of $B$ is either thin of order at least three in $X$, or a complex curve in $M$. Let $P_{1}, \ldots, P_{m}$ be points chosen from the complex components of $B$, and let $\tilde{\zeta}_{1}, \ldots, \tilde{\zeta}_{m}$ be holomorphic vector fields on $X$ such that $\tilde{\zeta}_{j}$ is not tangent to the leaf of the holomorphic foliation of $M$ at $P_{j}$. Then, replacing $\tilde{\xi}$ by $\tilde{\xi}+\varepsilon_{1} \tilde{\zeta}_{1}+\cdots+\varepsilon_{m} \tilde{\zeta}_{m}$ for some $\left(\varepsilon_{1}, \ldots, \varepsilon_{m}\right) \in \mathbb{C}^{m}$ if necessary, we may assume in advance that the set

$$
C=\left\{P \in M \mid(\tilde{\xi} \wedge \tilde{\eta})_{P}=0 \text { and } \tilde{\xi}_{P} \in T_{M}^{1,0}\right\}
$$


is finite and that the set

$$
D=\left\{P \in M \mid(\tilde{\xi} \wedge \tilde{\eta})_{P}=0 \text { and }(\operatorname{Re} \tilde{\xi})_{P} \in T_{M}\right\}
$$

is nowhere dense in the complex components of $B$. Here $T_{M}^{1,0}$ denotes the holomorphic tangent bundle of $M$.

We set

$$
\begin{aligned}
& Z_{1}=\pi\left(S_{(\alpha, \beta)}\right) \cap B \\
& Z_{2}=\left(\pi\left(S_{(\alpha, \beta)}\right) \backslash B\right) \cup\left(C \backslash \pi\left(S_{(\alpha, \beta)}\right)\right.
\end{aligned}
$$

and

$$
Z_{3}=\pi\left(S_{(\alpha, \beta)}\right) \cup B
$$

Then, for any $(\alpha, \beta)$ outside a set of Lebesgue measure zero, $\operatorname{Re}(\alpha \tilde{\xi}+\beta \tilde{\eta})$ is a Siu field for $M$ with respect to $Z_{j}(j=1,2,3)$.

If $(X, \omega)$ is flat, the existence of Siu fields is almost trivial. To illustrate this situation, we shall prove below an assertion which is stronger than Proposition 2.1 for complex tori.

Proposition 2.2. $\quad$ Let $(X, \omega)$ be a two dimensional flat Kähler manifold and let $M$ be a $C^{2}$-smooth compact real hypersurface in $X$. Then there exist a flat vector field $\xi$ and a $C^{1}$-smooth curve $Z \subset M$ such that $\xi$ is not tangential to $M$ at every point of $M \backslash Z$.

Proof. Let $P_{0} \in M$ be any point and let $x=\left(x_{1}, x_{2}, x_{3}, x_{4}\right)$ be a real local coordinate of $X$ around $P_{0}$ such that $x$ maps a neighbourhood of $P_{0}$ isometrically onto a neighbourhood of the origin in $\mathbb{R}^{4}$. We may assume that there exists a $C^{2}$ function $f$ on $U=\left\{x^{\prime}=\left(x_{1}, x_{2}, x_{3}\right)|| x_{j} \mid<1, j=1,2,3\right\}$ such that $M$ is defined by $x_{4}+f\left(x_{1}, x_{2}, x_{3}\right)=0$ in $\left\{x|| x_{j} \mid<1,1 \leq j \leq 4\right\}$.

Then we put $V\left(x^{\prime}\right)=\left(f_{x_{1}}, f_{x_{2}}, f_{x_{3}}, 1\right)$ and

$$
V_{0}\left(x^{\prime}\right)=\frac{V\left(x^{\prime}\right)}{\left\|V\left(x^{\prime}\right)\right\|} \in S^{3}:=\left\{x \in \mathbb{R}^{4} \mid\|x\|=1\right\} .
$$

Then $V_{0}$ is a $C^{1}$ map from $U$ to $S^{3}$.

Hence there exists a subset $A \subset S^{3}$ of Lebesgue measure zero such that, for all $\nu \in S^{3} \backslash A$, the preimage of $\nu^{\perp}=\left\{\nu^{\prime} \in S^{3} \mid \nu^{\prime} \perp \nu\right\}$ by $V_{0}$ is a $C^{1}$-smooth submanifold of $U$. The conclusion follows from this generic local existence immediately. 
From now on, let $(X, \omega)$ be a connected homogeneous Hermitian surface, let $m \in \mathbf{N}$, let $M \subset X$ be a connected $C^{m}$ Levi-flat, and let $\xi$ be a Siu field on $X$ for $M$. By taking a double cover of $X$ if necessary, we shall assume that $M$ bounds a domain $D$ in $X$.

For any nonnegative integers $p, q$ and $m$ we set

$$
\begin{aligned}
& L_{(2), m}^{p, q}(D, \xi)=\{u \mid u \text { is a measurable }(p, q) \text { form on } D \\
& \text { such that } \left.\left(\operatorname{Lie}_{\xi}\right)^{\nu} u \text { are square integrable for all } 0 \leq \nu \leq m\right\}
\end{aligned}
$$

and

$$
\|u\|_{m}^{2}=\sum_{\nu=0}^{m}\left\|\left(\operatorname{Lie}_{\xi}\right)^{\nu} u\right\|^{2}
$$

for any $u \in L_{(2), m}^{p, q}(D, \xi)$. Here $\operatorname{Lie}_{\xi}$ denotes the Lie derivative with respect to $\xi$.

By $(,)_{m}$ we denote the inner product associated to \|\|$_{m}$, and by $\bar{\partial}_{m}^{*}$ the adjoint of $\bar{\partial}$ with respect to \|\|$_{m}$.

Proposition 2.3. Let $P_{0} \in M$ be any point where $\xi$ is not tangential to $M$. Then there exists a neighbourhood $U$ of $P_{0}$ in $X$ such that the following holds.

$\left\{g \in L_{(2), m}^{p, q+1}(D \cap U) \mid\right.$ there exists an $f \in L_{(2), m}^{p, q}(D \cap U)$ such that $(\bar{\partial} u, g)_{m}=$ $(u, f)_{m}$ holds for every compactly supported $C^{\infty}(p, q)$ form $u$ on $\left.U\right\}=$ $\left\{g \in L_{(2), m}^{p, q+1}(D \cap U) \mid\left(\operatorname{Lie}_{\xi}\right)^{\nu} g \in\right.$ Dom $\bar{\partial}^{*}$ for all $\left.0 \leq \nu \leq m\right\}$.

For the proof see Lemma 4.3 in $[\mathrm{S}-2]$.

In virtue of a removability result for singularities along thin subsets (cf. Lemma 3.2 in [S-2]), we obtain from Proposition 2.3 the following basic property of the domain Dom $\bar{\partial}_{m}^{*}$ of $\bar{\partial}_{m}^{*}$.

Proposition 2.4 ([S-2, Proposition 5.1]).

$$
\operatorname{Dom} \bar{\partial}_{m}^{*}=\left\{u \in L_{(2)}^{p, q}(D) \mid\left(\operatorname{Lie}_{\xi}\right)^{\nu} u \in \operatorname{Dom} \bar{\partial}^{*} \quad \text { for all } 0 \leq \nu \leq m\right\} .
$$

We shall finally rely on the following.

Proposition 2.5 (cf. Proposition 6.4 in [S-2]). Let $m \geq 4$ and let $u$ be a $C^{\infty}$ function on $D$ such that $\bar{\partial} u$ is of class $C^{m-1}$ on $\bar{D}$ and that $\left\|\xi^{j} u\right\|<\infty$ for $0 \leq j \leq m-1$. Then every derivative of $u$ of order up to $m-1$ is locally square integrable on $\bar{D}$. 


\section{$\S 3 . \quad$ Proof of Theorem 0.1}

Let $(X, \omega)$ be a connected homogeneous Kähler surface. In virtue of the works of Shimizu [Sh] and Dorfmeister-Nakajima $[\mathrm{D}-\mathrm{N}],(X, \omega)$ is one of the following.

1) $\left(\mathbb{P}^{2}\right.$, const $\cdot$ Fubini-Study form $)$

2) Quotients of $\left(\mathbb{C}^{2}, i\left(d z_{1} \wedge d \bar{z}_{1}+d z_{2} \wedge d \bar{z}_{2}\right)\right)$ by the standard action of a discrete subgroup of $\mathbb{C}^{2}$

3) $\left(X_{1} \times X_{2}, \pi_{1}^{*} \omega_{1}+\pi_{2}^{*} \omega_{2}\right)$, where $\left(X_{j}, \omega_{j}\right)$ are one dimensional homogeneous Kähler manifolds and $\pi_{j}: X_{1} \times X_{2} \longrightarrow X_{j}$ denote the projections.

If $X$ is noncompact, then $X$ is weakly 1-complete (i.e. $X$ admits a $C^{\infty}$ plurisubharmonic exhaustion function, $\operatorname{say} \varphi$ ) in view of the above classification.

Therefore, if $M \subset X$ were a $C^{5}$ Levi-flat such that there exists a function $\varrho$ on $X \backslash M$ satisfying (0.1) and (0.2), then $X \backslash M$ would admit a $C^{2}$ plurisubharmonic exhaustion function $\varphi-\log (-\varrho)$ which we call $\psi$. By smoothing $-\log (-\varrho)$ if necessary, we may assume that $\psi$ is $C^{\infty}$ and strictly plurisubharmonic near $M$.

On the other hand, since $(X, \omega)$ is homogeneous, the canonical bundle $K_{X}$ admits a fiber metric $h$ whose curvature form is everywhere seminegative.

Therefore, by a standard argument of solving the $\bar{\partial}$-equation by exploiting the seminegativity of $K_{X}$ and the positivity of $\partial \bar{\partial} \psi$ near $M$, one can produce holomorphic functions on $X \backslash M$ that separate the points near $M$.

Being homogeneous, $X$ contains no exceptional curves (i.e. there exists no compact connected complex analytic subset of dimension one in $X$ which is contractible to a point analytically).

Hence one can find holomorphic functions on $X \backslash M$, say $f_{1}, \ldots, f_{m}$, such that $\psi+\sum_{k=1}^{m}\left|f_{k}\right|^{2}$ is a strictly plurisubharmonic exhaustion function on $X \backslash M$, so that $X \backslash M$ is a Stein manifold by a theorem of H. Grauert [G].

However, at least one component of $X \backslash M$ has at least two ends, since $X$ is noncompact. This is an absurdity because $X \backslash M$ is a Stein manifold of pure dimension two.

If $X$ is compact, we argue as follows: Let $M \subset X$ be as above. We may assume that $M$ bounds a domain $D \subset X$. We shall show that the space of holomorphic sections of the canonical bundle $K_{X}$ over $X$ is dense in $L_{(2)}^{2,0}(D) \cap$ Ker $\bar{\partial}$, which is an absurdity, for $\operatorname{dim} H^{0}\left(X, K_{X}\right)<\infty$ because $X$ is compact, and $\operatorname{dim}\left(L_{(2)}^{2,0}(D) \cap \operatorname{Ker} \bar{\partial}\right)=\infty$ because by the assumption (0.1) $D$ is known to be hyperconvex (cf. [O-S]). 
To deduce this absurdity, first we note that the condition (0.1) implies that there exists $c^{\prime}>0$ such that

$$
-i \partial \bar{\partial} \log (-\varrho)>\frac{c}{2} \omega+i c^{\prime} \varrho^{-2} \partial \varrho \wedge \bar{\partial} \varrho
$$

(cf. $[\mathrm{O}-\mathrm{S}]$ ). From this inequality it is easy to show that the bounded exhaustion function

$$
\psi(x)=-\varepsilon / \log (-\log (-\varrho(x))+C))
$$

satisfies $\inf \psi>-1$ and $i \partial \bar{\partial} \psi>2 i \partial \psi \wedge \bar{\partial} \psi$ on $D$ for a sufficiently small positive $\varepsilon$. Here $C$ is a constant satisfying $-\varrho<e^{C}$ on $D$.

Let $f \in L_{(2)}^{2,0}(D) \cap \operatorname{Ker} \bar{\partial}$, and put

$$
v_{k}=f \wedge \bar{\partial} \chi(k \psi) .
$$

Here $\chi$ is defined as in $\S 1$.

Then, for any Siu field $\xi$ for $M$ on $X$ and for any $u \in L_{(2), 4}^{2,1}(D, \xi) \cap \operatorname{Dom} \bar{\partial} \cap$ Dom $\bar{\partial}_{4}^{*}$,

$$
\begin{aligned}
\left|\left(u, v_{k}\right)\right|_{4}^{2} \leq & 2(i \partial \bar{\partial} \psi \Lambda u, u)\left((i \partial \bar{\partial} \psi \Lambda)^{-1} v_{k}, v_{k}\right) \\
& +2 \sum_{\nu=1}^{4}\left|\left(\left(\operatorname{Lie}_{\xi}\right)^{\nu} u,\left(\operatorname{Lie}_{\xi}\right)^{\nu} v_{k}\right)\right|^{2}
\end{aligned}
$$

Hence, for any $k$ one can choose a Siu field $\xi$ in such a way that

$$
\begin{aligned}
\left|\left(u, v_{k}\right)_{4}\right|^{2} \leq & 2(i \partial \bar{\partial} \psi \Lambda u, u)\left((i \partial \bar{\partial} \psi \Lambda)^{-1} v_{k}, v_{k}\right) \\
& +\frac{1}{k} \sum_{\nu=1}^{4}\left(i \partial \bar{\partial} \psi \Lambda\left(\operatorname{Lie}_{\xi}\right)^{\nu} u,\left(\operatorname{Lie}_{\xi}\right)^{\nu} u\right)
\end{aligned}
$$

holds true.

Therefore, combining this estimate with Propositions 1.1 and 2.4 we obtain

$$
\left|\left(u, v_{k}\right)_{4}\right|^{2} \leq 3\left((i \partial \bar{\partial} \psi \Lambda)^{-1} v_{k}, v_{k}\right)\left(\|\bar{\partial} u\|_{4}^{2}+\left\|\bar{\partial}^{*} u\right\|_{4}^{2}\right) .
$$

Clearly $\left((i \partial \bar{\partial} \psi \Lambda)^{-1} v_{k}, v_{k}\right) \longrightarrow 0$ as $k \longrightarrow \infty$. Hence one can find a sequence $\xi_{k}$ of Siu fields and $f_{k} \in L_{(2), 4}^{2,0}\left(D, \xi_{k}\right)$ such that $\bar{\partial} f_{k}=v_{k}$ and $\lim _{k \rightarrow \infty}\left\|f_{k}\right\|=0$. Therefore, by Proposition 2.5 and by Sobolev's embedding theorem we obtain a sequence $\tilde{f}_{k}=\chi(k \psi) f-f_{k}$ converging to $f$ in $L_{(2)}^{2,0}(D)$ which are of class $C^{1}$ on $\bar{D}$.

Next we exploit (3.1) on $X \backslash \bar{D}$ to obtain holomorphic extensions of $\tilde{f}_{k}$ to $X$. 
For that, let $\hat{f}_{k}$ be a $C^{1}(2,0)$ form on $X$ extending $\tilde{f}_{k}$, and put $w_{k}=\bar{\partial} \hat{f}_{k}$. Since $K_{X}$ is seminegative by the homogeneity of $X$, for any $w_{k}$ one can find a $(2,0)$ form $g_{k}$ on $X \backslash \bar{D}$ such that

$$
\int_{X \backslash \bar{D}}(-\varrho)^{-1}\left|g_{k}\right|^{2} d V \leq C^{\prime} \int_{X \backslash \bar{D}}(-\varrho)^{-1}\left|w_{k}\right|^{2} d V
$$

holds. Here $C^{\prime}$ is a constant independent of $k$.

Clearly $\hat{f}_{k}-g_{k}$ is holomorphic on $X \backslash \bar{D}$ and holomorphically extends to an element $F_{k}$ of $H^{0}\left(X, K_{X}\right)$ such that $F_{k} \mid D=\tilde{f}_{k}$.

Therefore $H^{0}\left(X, K_{X}\right)$ is dense in $L_{(2)}^{2,0}(D) \cap$ Ker $\bar{\partial}$ which was the desired contradiction.

Before going into the proof of Proposition 0.1, let us recall a Lemma from $[\mathrm{M}-\mathrm{O}]$.

Lemma 3.1 ([M-O]). Let $C$ be a complex hypersurface in $\mathbb{C}^{2}$ defined by $C=\{(t, f(t)) \mid t \in V\}$ for some open $V \subset \mathbb{C}$ and holomorphic $f$. Then for any $p \in C$ there exists a neighbourhood $U \ni p$ in $\mathbb{C}^{2}$ such that

$$
\begin{aligned}
& \sum_{j, k=1}^{2} \frac{\partial^{2}\left(-\log \delta_{C}\right)}{\partial z_{j} \partial \bar{z}_{k}}\left(z_{1}, z_{2}\right) \xi_{j} \bar{\xi}_{k} \\
& =\left.\frac{\left|\partial^{2} f / \partial t^{2}\right|^{2}\left|\xi_{1}+\xi_{2} \partial f / \partial t\right|^{2}}{2\left(|\partial f / \partial t|^{2}+1\right)^{2}\left(|\partial f / \partial t|^{2}+1\right)^{2}-\left|\partial^{2} f / \partial t^{2}\right|^{2}\left|z_{2}-f(t)\right|^{2}}\right|_{t=t\left(z_{1}, z_{2}\right)}
\end{aligned}
$$

for any $\left(z_{1}, z_{2}\right) \in U \backslash C$ and $\left(\xi_{1}, \xi_{2}\right) \in \mathbb{C}^{2}$. Here $\delta_{C}=\delta_{C}\left(z_{1}, z_{2}\right)$ denotes the Euclidean distance from $\left(z_{1}, z_{2}\right)$ to $C$ and $t\left(z_{1}, z_{2}\right)$ is the solution of $z_{1}-t+$ $\left\{z_{2}-f(t)\right\} \partial \bar{f} / \partial \bar{t}=0$.

Proof of Proposition 0.1. Let $(X, \omega)$ be a flat complex torus of dimension two and let $M \subset X$ be a $C^{5}$ Levi-flat.

Suppose that $M$ does not contain any complex line segment, in the sense that the preimage of $M$ in the universal cover $\mathbb{C}^{2}$ of $X$ does not contain any nonempty open subset of a complex line in $\mathbb{C}^{2}$.

Then the set of inflection points of the leaves of the holomorphic foliation of $M$, say $S$, is locally the graph of a solution to the equation $f^{m}+a_{1}(t) f^{m-1}+$ $\cdots+a_{m}(t)=0$ for some $C^{5}$ functions $a_{k}(t)$ of real one variable $t$.

Hence $S$ admits a Stein neighbourhood system in $X$.

On the other hand, from the above lemma, it is easy to see that, for any neighbourhood $W$ of $S$ in $X$, one can find a neighbourhood $U \supset M$ in $X$ such that $-\log \delta_{M}$ is strictly plurisubharmonic on $U \backslash M \backslash W$. Here $\delta_{M}$ denotes the distance to $M$ with respect to $\omega$. 
Therefore, taking any $C^{\infty}$ real valued function $\psi$ on $X$ which is strictly plurisubharmonic on a neighbourhood of $S$, one can find a neighbourhood $U_{0} \supset$ $M$ and a positive number $\varepsilon$ such that $i \partial \bar{\partial}\left(\varepsilon \psi-\log \delta_{M}\right)>\varepsilon^{2} \omega$ holds true on $U_{0} \backslash M$.

Hence, after smoothing $-\delta_{M} e^{-\varepsilon \psi}$ outside a neighbourhood of $M$ if necessary, we obtain a $C^{2}$ function $\varrho$ on $X \backslash M$ satisfying the conditions (0.1) and (0.2), which of course contradicts Theorem 0.1 .

Thus $M$ must contain a complex line segment.

\section{$\S 4$. Levi Scrolls}

Let us introduce a class of Levi-flat $C R$ three-folds which are fibered over compact Riemann surfaces by a $C R$ map allowing certain singular fibers.

Definition. A Levi scroll is a real analytic 3-dimensional compact Levi flat $C R$ manifold $L$ equipped with a surjective $C R$ map $\hat{\pi}$ onto a compact Riemann surface $C$ such that the critical set of $\hat{\pi}$ is the union of finitely many compact complex curves. We call $L$ a Levi scroll over $C$, the compact complex curves in $L$ the critical fibers, and $C$ the base of $L$.

It is easy to see that the complex curves in a Levi flat 3-manifold are nonsingular. In particular, all the critical fibers of a Levi scroll are compact Riemann surfaces.

Example. Let $\hat{\mathbb{C}}=\mathbb{C} \cup\{\infty\}$ be the Riemann sphere with inhomogeneous coordinate $\zeta$ and let $E$ be the quotient of $\mathbb{C}^{*}=\mathbb{C} \backslash\{0\}$ by the action $z \longrightarrow 2^{m} z(m \in \mathbf{Z})$. We put

$$
L_{0}=\left\{(\zeta, z) \in \hat{\mathbb{C}} \times \mathbb{C}^{*} \mid \operatorname{Im}(\zeta \exp (2 \pi i \log z / \log 2)=0\}\right.
$$

and let $L_{0}$ be the image of $\hat{L}_{0}$ by the natural projection from $\mathbb{C} \times \mathbb{C}^{*}$ onto $\mathbb{C} \times E$. Let $L_{1}$ be the closure of $\hat{L}_{0}$ in $\hat{\mathbb{C}} \times E$. Then $L_{1}$ is a Levi-flat and mapped onto $\hat{\mathbb{C}}$ by the projection $p_{1}$ from $\hat{\mathbb{C}} \times E$ onto its first factor $\hat{\mathbb{C}}$.

Clearly the compact complex curves contained in $L_{1}$ are $p_{1}^{-1}(0)$ and $p_{1}^{-1}(\infty)$.

Hence $L_{1}$ is a Levi scroll over $\hat{\mathbb{C}}$.

This example arose first in [O-2] as the boundary of a Stein domain which has a product structure. In fact, $L_{1}$ bounds a domain which is equivalent to $\mathbb{C}^{*} \times\{z \in \mathbb{C}|1<| z \mid<\sqrt{2}\}$. (See also [Nem].) 
The following describes real analytic Levi-flats which are Levi scrolls over compact Riemann surfaces with respect to the restriction of the bundle projection with elliptic curves as fibers.

Proposition 4.1. Let $E=\mathbb{C} /(\mathbf{Z}+\tau \mathbf{Z})(\operatorname{Im} \tau>0)$, let $C$ be a compact Riemann surface, let $\sigma: H_{1}(C, \mathbf{Z}) \longrightarrow E$ be a homomorphism, and let $\tilde{\pi}: \mathcal{E} \longrightarrow$ $C$ be the E-bundle associated to $\sigma$. Then the Levi scrolls in $\mathcal{E}$ over $C$ with respect to the restriction of $\tilde{\pi}$ are, up to the parallel translate along the fibers of $\mathcal{E}$, in one to one correspondence with the pairs $(G, l)$ of nonzero meromorphic 1-forms $G$ on $C$ and 1-dimensional closed subgroups $l \subset E$ satisfying

i) Poles of $G$ are of order one, and the residues $\operatorname{Res}(G, P)$ are all contained in $l$ for $P \in C$.

ii) The kernel of the canonical homomorphism from $i \operatorname{Res}(G, P) \cdot \mathbb{R}$ to $E / l$ is $(i \operatorname{Res}(G, P) \mathbf{Z}+\mathbf{Z}+\tau \mathbf{Z}+l) \cap i \operatorname{Res}(G, P) \cdot \mathbb{R}$

iii) For any $\gamma \in H_{1}(C, \mathbf{Z}), \int_{\gamma} G \subset l+\sigma(\gamma)$. Here $\int_{\gamma} G$ denotes the set $\left\{\int_{\Gamma} G \mid \Gamma \in\right.$ $\gamma$ and $\Gamma$ does not pass through any pole of $G\}$.

Proof. Let $L \subset \mathcal{E}$ be a Levi scroll over $C$ with critical fibers $\pi^{-1}\left(P_{j}\right)$.

We put

$$
\begin{aligned}
C^{\prime} & =C \backslash\left\{P_{1}, \ldots, P_{n}\right\} \\
L^{\prime} & =L \backslash \pi^{-1}\left(\left\{P_{1}, \ldots, P_{n}\right\}\right)
\end{aligned}
$$

and

$$
\mathcal{E}^{\prime}=\mathcal{E} \backslash \pi^{-1}\left(\left\{P_{1}, \ldots, P_{n}\right\}\right)
$$

Then $\pi$ induces a locally hyperconvex Stein submersion from $\mathcal{E} \backslash L\left(=\mathcal{E}^{\prime} \backslash L^{\prime}\right)$ onto $C^{\prime}$. Namely, for any point $P \in C^{\prime}$, there exist a neighbourhood $U \ni P$ and a strictly plurisubharmonic function $\varphi: \pi^{-1}(U) \longrightarrow[-1,0)$ such that $\pi \mid \varphi^{-1}([-1, c))$ is proper for every $c<0$.

Hence $\mathcal{E} \backslash L$ is a Stein manifold (cf. [B] for instance).

By the real analyticity of $L$, there exist a neighbourhood $W \supset L$ and a complex analytic foliation $\mathscr{F}$ on $W$ extending the holomorphic foliation on $L$. Since $\mathcal{E} \backslash L$ is Stein, $\mathscr{F}$ extends further to $\mathcal{E}$ as a foliation with finitely many singularities, since any holomorphic section of the projectivization of $T^{1,0} \mathcal{E}$, defined over $W$, extends to $\mathcal{E}$ as a meromorphic section by a theorem of S. Ivashkovitch [Iv]. Let us denote this meromorphic section by $\beta$. 
Let $U_{j}$ be a neighbourhood of $P_{j}$ such that $\pi^{-1}\left(U_{j}\right) \simeq U_{j} \times E$. Then $\beta \mid \pi^{-1}\left(P_{j}\right)$ is naturally identified with a constant map. Therefore $\beta \mid \pi^{-1}\left(U_{j}\right)$ factors through a holomorphic map say $\beta_{j}$ on $U_{j}$, by shrinking $U_{j}$ if necessary.

Hence $\beta$ is constant fiberwise. In particular $\beta$ has no points of indeterminancy, so that $\mathscr{F}$ actually extends to a holomorphic foliation $\tilde{\mathscr{F}}$ on $\mathcal{E}$ without any singularities.

For each $j$, we choose holomorphic local coordinates around $P_{j}$ and $\beta\left(P_{j}\right)$ so that $\beta_{j}=\beta_{j}(z)$ is of the form $z^{k_{j}}$ for some $k_{j} \in N$.

Since $L$ is a Levi-flat, it is defined on a neighbourhood of $\pi^{-1}\left(P_{j}\right)$ by $\operatorname{Re}\left(z \exp h_{j}(\zeta)\right)=0$ up to the terms of order at least 2 in $z$ and $\bar{z}$, for some nonconstant (locally well defined) harmonic function $h_{j}$ on $E$. From this it is easy to see that $k_{j}=1$ for all $j$.

Let $P \in C^{\prime}$ be any point. Then there exist a neighbourhood $U \ni P$ and a biholomorphic automorphism $\chi$ of $\pi^{-1}(U)$ over $U$ which transforms the leaves of the foliation $\tilde{\mathscr{F}}$ to the flat sections of $\mathcal{E}$ over $U$, by fiberwise parallel translation. We fix $\chi$ in such a way that $\chi \mid \pi^{-1}(P)=i d$.

Let $\mu: \tilde{C}^{\prime} \longrightarrow C^{\prime}$ be the universal cover of $C^{\prime}$. Then the fiber component of $\chi$ continues analytically to $\tilde{C}^{\prime}$ as a holomorphic map to $E$, say $\tilde{\alpha}: \tilde{C}^{\prime} \longrightarrow E$.

Then $d \tilde{\alpha}$ descends as a map from $T^{1,0}\left(C^{\prime}\right)$ and $\beta \mid C^{\prime}$ is expressed as the projectivization of the map

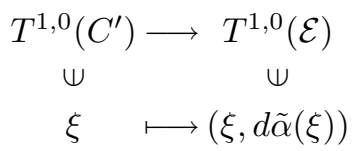

Clearly, as a 1-form on $C, d \tilde{\alpha}$ has poles of order one at $P_{1}, \ldots, P_{n}$.

Let $\tilde{P}$ be any point of $L \cap \tilde{\pi}^{-1}(P)$, and let $l=L \cap \tilde{\pi}^{-1}(P)-\tilde{P}$, where the subtraction is with respect to the additive structure of $E$. Then iii) is satisfied because parallel translations defined by the values of $\tilde{\alpha}$ along closed paths in $C^{\prime} \operatorname{map} L \cap \tilde{\pi}^{-1}(P)$ into itself.

Thus we obtain a pair $(d \tilde{\alpha}, l)$ satisfying i)-iii).

Conversely, if a pair $(G, l)$ is given to satisfy i)-iii), let $P_{1}, \ldots, P_{n}$ be the poles of $G$, and let $C^{\prime}, \mathcal{E}^{\prime}, \tilde{C}^{\prime}$ be as above. Then, for any point $\tilde{P} \in \tilde{C}^{\prime}$, the subset $L(G, l) \subset \tilde{C}^{\prime} \times E$ defined by

$$
L(G, l)=\bigcup_{\tilde{Q} \in \tilde{C}^{\prime}}\left(\tilde{Q}, l+\int_{\tilde{P}}^{\tilde{Q}} G\right)
$$

descends by iii) to a closed $C^{\omega}$ real hypersurface of $\mathcal{E}^{\prime}$. By i) and ii), its closure is a smooth hypersurface of $\mathcal{E}$, which is a Levi scroll over $C$ with critical fibers 
$\tilde{\pi}^{-1}\left(P_{1}\right), \ldots, \tilde{\pi}^{-1}\left(P_{n}\right)$. Clearly, this construction is the inverse of the former correspondence between $L$ and $(G, l)$ up to the parallel displacements of $L$ in the fiber direction.

\section{$\S 5 . \quad$ Classification of Levi-flats in Tori}

Based on the results in the previous sections, we shall prove Theorems 0.2 and 0.3 .

Definition. We say a Levi-flat $M$ in a complex torus $X$ holomorphically flat if the preimage of $M$ by the universal covering map $\mathbb{C}^{\mathrm{n}} \longrightarrow X$ is a union of complex affine hypersurfaces of $\mathbb{C}^{\mathrm{n}}$.

Proof of Theorem 0.2. Let $M$ be a real analytic Levi-flat in a complex torus $X$ of dimension two. By Proposition 0.1, $M$ contains a complex line segment.

If there passes a complex line segment through every point of $M$, then $M$ is holomorphically flat by definition. If not, $M$ does not contain any complex line segment which continues analytically to a dense leaf of the holomorphic foliation.

Let $A$ be the union of complex line segments in $M$. Then the components of $A$ are all elliptic curves and there exists a surjective holomorphic map $f$ with connected fibers from $X$ onto another elliptic curve say $C$, such that the connected components of $A$ are mapped to points.

If the number of the connected components of $A$ is finite, then it follows similarly as in the proof of Proposition 4.1 that $M$ is a Levi scroll over $C$.

Suppose on the contrary that the number of the connected components of $A$ were infinite.

Then there would exist a sequence of distinct elliptic curves $E_{\mu} \subset A$ converging to some elliptic curve $E \subset M$.

Since the normal bundle of $E$ is trivial and $X$ is a complex torus, for any tubular neighbourhood $U \supset E$ in $X$, every component of $U \backslash M$ contains an analytic family of elliptic curves as parallel translates of $E$ in the normal direction.

Therefore, since $X \backslash M$ is pseudoconvex, by Nishino's theorem [N], $X \backslash M$ is the union of elliptic curves and accordingly so is $M$.

But this is a case which has already been excluded. Therefore $A$ has only finitely many connected components, so that $M$ is a Levi scroll.

Proof of Theorem 0.3. If a two dimensional complex torus contains a Levi scroll, then its critical fibers must be elliptic curves because of an obvious 
topological reason. Therefore the torus must be holomorphically fibered over an elliptic curve. The converse is clear from Proposition 4.1 and the classical existence theorem for meromorphic forms on compact Riemann surfaces (e.g. the Riemann-Roch theorem).

Notes. By applying the method in [S-1] instead of [S-2], one can show that any $C^{\infty}$ Levi-flat in a complex torus of dimension $n \geq 3$ contains a complex $(n-1)$-plane segment. Based on this fact, the classification of $C^{\omega}$ Levi-flats in tori can be done similarly. The detail of this extended classification theory will appear elsewhere. As for the Levi-flats in other complex surfaces, many questions seems to be left open. It might be worthwhile to classify the Leviflats in K3 surfaces because it will need basic information on the holomorphic foliations on them.

\section{References}

[B-C] Berndtsson, B. and Charpentier, P., A Sobolev mapping property of the Bergman kernel, Math. Z., 235 (2000), 1-10.

[B] Brun, J., Sur le problème de Levi dans certain fibrés, Manuscripta Math., 14 (1974), 217-222.

[C] Cerveau, D., Minimaux des feuilletages algébriques de $\mathbb{C P}^{n}$, Ann. Inst. Fourier, 43 (1993), 1535-1543.

[D-1] Demailly, J.-P., Estimation $L^{2}$ pour l'opérateur $\bar{\partial}$ d'un fibré vectoriel holomorphe sémipositif au dessus d'une variété kählerienne complète, Ann. Sci. École Norm. Sup., 15 (1982), 457-511.

[D-2] $\longrightarrow$, Cohomology of $q$-convex spaces in top degrees, Math. Z., 204 (1990), 283295.

[D-N] Dorfmeister, J. and Nakajima, K., The fundamental conjecture for homogeneous Kähler manifolds, Acta Math., 161 (1988), 23-70.

[G-W] Greene, R. E. and Wu, H., Embedding of open Riemannian manifolds by harmonic functions, Ann. Inst. Fourier, 25 (1975), 215-235.

[H-I] Henkin, G. M. and Iordan, A., Regularity of $\bar{\partial}$ on pseudoconcave compacts and applications, Asian J. Math., 4 (2000), 855-884, Erratum, Asian J. Math., 7 (2003), 147-148.

[H-1] Hörmander, L., $L^{2}$ estimates and existence theorems for the $\bar{\partial}$-operator, Acta Math., 113 (1965), 89-152.

[H-2] Complex Analysis in Several Variables, Van Nostrand, 1966.

[I] Iordan, A., On the non-existence of smooth Levi-flat hypersurfaces in $\mathbb{C P}_{n}, A d v$. Stud. Pure Math., 42 (2004), 123-126.

[Iv] Ivashkovitch, S., The Hartogs-type extension theorem for meromorphic maps into compact Kähler manifolds, Invent. Math., 109 (1992), 47-54.

[LN] LinsNeto, A., A note on projective Levi flats and minimal sets of algebraic foliations, Ann. Inst. Fourier, 49 (1999), 1369-1385.

[M-O] Matsumoto, K. and Ohsawa, T., On the real analytic Levi flat hypersurfaces in complex tori of dimension two, Ann. Inst. Fourier, 52 (2002), 1525-1532.

[Nem] Nemirovski, S., Stein domains with Levi-flat boundaries on compact complex surfaces, Math. Notes, 66 (1999), 522-525. 
[N] Nishino, T., L'existence d'une fonction analytique sur une variété analytique complexe a deux dimension, Publ. RIMS, Kyoto Univ., 18 (1982), 387-419.

[O-1] Ohsawa, T., On complete Kähler domains with $C^{1}$-boundary, Publ. RIMS, Kyoto Univ., 16 (1980), 929-940.

[O-2] A Stein domain with smooth boundary which has a product structure, Publ. RIMS, Kyoto Univ., 18 (1982), 1185-1186.

[O-3] Completeness of noncompact analytic spaces, Publ. RIMS, Kyoto Univ., 20 (1984), 683-692.

[O-4] Vanishing theorems on complete Kähler manifolds, Publ. RIMS, Kyoto Univ., 20 (1984), 21-38.

[O-5] On the extension of $L^{2}$ holomorphic functions $V$-effects of generalization, Nagoya Math. J., 161 (2001), 1-21.

[O-6] On a curvature condition that implies a cohomology injectivity theorem of Kollár-Skoda type, Publ. RIMS, Kyoto Univ., 41 (2005), 565-577.

[O-S] Ohsawa, T. and Sibony, N., Bounded P.S.H. functions and pseudoconvexity in Kähler manifold, Nagoya Math. J., 149 (1996), 1-8.

[Sh] Shimizu, S., Homogeneous Kähler manifolds of complex dimension two, Tohoku Math. J., 34 (1982), 53-63.

[S-1] Siu, Y.-T., Nonexistence of smooth Levi-flat hypersurfaces in complex projective spaces of dimension $\geq 3$, Ann. of Math., 151 (2000), 1217-1243.

[S-2], $\bar{\partial}$-regularity for weakly pseudoconvex domains in compact Hermitian spaces with respect to invariant metrics, Ann. of Math., 156 (2002), 595-621.

[T] Takeuchi, A., Domaines pseudoconvexes infinis et la métrique riemanniennes dans un espace projectif, J. Math. Soc. Japan, 16 (1964), 159-181. 